\title{
Organismos vivos: pilas voltaicas vivientes Una discusión sobre la evolución conceptual de la bioquímica
}

\author{
Lucy Rivera Rojas \\ Departamento de Bioquímica y Nutrición. Facultad de Ciencias. Pontificia Universidad Javeriana. \\ Correspondencia: lucy.rivera@javeriana.edu.co
}

Recibido: 09-08-06 / Aceptado: 06-09-06

\begin{abstract}
Resumen
Los organismos pueden ser clasificados en fotótrofos, quimiorganótrofos y quimiolitótrofos de acuerdo a las formas como obtienen energía. Pueden utilizar como aceptor final de electrones bien sea el oxígeno o cualquier otra molécula que esté en capacidad de cumplir la misma función. A pesar de las diferencias metabólicas que presentan estos organismos; la forma como obtienen energía se rige bajo un principio físico básico: emplean un sustrato que está en capacidad de generar un flujo de electrones termodinámicamente favorable, en donde es liberada la energía empleada en realizar múltiples funciones. En este sentido, los organismos son verdaderas pilas voltaicas vivientes. Estas conclusiones han hecho evolucionar la estructura conceptual de la bioquímica; sin embargo, el sistema educativo no ha evolucionado a la par. La discusión presenta la importancia de re-escribir los libros de texto de bioquímica en los que sean plasmados los nuevos conceptos que emergen gracias a los hallazgos de formas de vida consideradas hasta hace poco inimaginables.

Palabras claves: bioquímica, enseñanza, fotótrofos, metabolismo energético, pilas voltáicas, quimiolitótrofos, quimiorganótrofos.
\end{abstract}

\begin{abstract}
Organisms can be classified as phototroph, chemorganotroph and chemolithotroph, according to the way they obtain energy Oxygen or any other molecule can be used as electron acceptor as long as it exhibits the same function. In spite of the metabolic differences, these organisms are governed by a basic physical principle: substrates are used to generate a flow of electrons thermodynamically favorable, where the produced energy can be used for multiple functions. In this sense, it can be said that organisms are true voltaic batteries. This type of assumptions have made a considerable advance in the biochemical conceptual structure. However, the educational system has not change at the same time. The present discussion demonstrates the importance of re-writing biochemistry text books in order to capture the new perceptions that emerge from the discoveries of new types of life considered unimaginable until recently,
\end{abstract}

Key words: chemolithotrophs, chemorganotrophs, energy metabolism, phototrophs, voltaic battery, teaching. 
Cualquier organismo debe asegurar mecanismos que le permitan proveerse de energía para poder realizar las múltiples funciones que lo caracterizan como ser vivo. Los organismos más ampliamente distribuidos en el planeta Tierra, y así los más ampliamente conocidos, son aquellos que logran extraer energía bien sea de moléculas orgánicas o a partir de la radiación solar. Existe adicionalmente una amplia variedad de organismos, todos ellos del mundo microscópico, capaces de extraer energía de fuentes que para la mayoría de las especies seria absolutamente imposible hacerlo. Aunque han permanecido también en el planeta por millones de años, sólo en las últimas décadas ha sido relevante su estudio; esto gracias a la gran cantidad de aplicaciones industriales, comerciales y ecológicas que recientemente se les ha encontrado.

Según la fuente de energía, los organismos son clasificados en fotótrofos si obtienen energía de la radiación solar, o bien quimiótrofos, si su fuente de energía es de naturaleza química. Dentro de los quimiótrofos es posible establecer dos categorías; los quimiorganótrofos, quienes obtienen energía a partir de moléculas orgánicas, y los quimiolitótrofos, aquellos que obtienen energía a partir de moléculas inorgánicas (1).

La vida que se conoce es fundamentalmente fotótrofa o quimiorganótrofa. Dentro de los organismos fotótrofos se encuentran bacterias, algas y todas las plantas. Dentro de los quimiorganótrofos se encuentra una amplia variedad de bacterias, hongos, levaduras, protozoos y por supuesto todos los animales. Un poco menos conocido, pero no menos importante en el mantenimiento del ecosistema, es el sistema de vida quimiolitótrofo; esto es, la capacidad que tienen ciertos organismos de los dominios bacteria y archaea de extraer energía de sustancias inorgánicas como azufre elemental $\left(\mathrm{S}^{\circ}\right)$, sulfuro de hidrógeno $\left(\mathrm{H}_{2} \mathrm{~S}\right)$, iones ferrosos $\left(\mathrm{Fe}^{2+}\right)$, pirita $\left(\mathrm{FeS}_{2}\right)$, amoniaco $\left(\mathrm{NH}_{3}\right)$, nitritos $\left(\mathrm{NO}_{2}^{-}\right)$e hidrógeno molecular $\left(\mathrm{H}_{2}\right)$.

El estudio del metabolismo energético de los quimiolitótrofos resulta impactante por cuanto son organismos que logran sobrevivir en condiciones consideradas desde el punto de vista humano, extremas. Muchos de estos organismos viven en ambientes con carencia total de sustratos orgánicos, a pH inferiores a 2,0 y a temperaturas cercanas a $\operatorname{los} 100^{\circ} \mathrm{C}$. Es el caso de la archaea Sulfolobus acidocaldarius que se encuentra en hábitats volcánicos, en fuentes hidrotermales ácidas y calientes o en fuentes termales de azufre como en el parque Yelowstone en Estados Unidos. Esta archaea extrae toda la energía necesaria para su metabolismo de la oxidación del sulfuro de hidrógeno y del azufre elemental. Thiobacillus ferrooxidans es una bacteria que extrae energía a partir de los iones ferrosos y de la pirita; su habitat se encuentra en las minas de carbón en proceso de explotación y en las aguas ácidas resultantes. Las bacterias nitrificantes obtienen energía de la oxidación del amoniaco y de los nitritos resultantes de la descomposición del material orgánico proveniente de residuos de plantas y animales principalmente. Las archaeas metanogénicas habitan los pantanos y pueden obtener energía a partir de la oxidación del hidrógeno (1).

Lo asombroso de todo ello, radica en que todos estos organismos-fotótrofos, quimiorganótrofos y quimiolitótrofos, a pesar de su gran diversidad genética, metabólica y de hábitat; utilizan el mismo principio físico básico para la obtención de energía: la transferencia de electrones desde un donador con un potencial de óxidoreducción muy negativo (baja afinidad por los electrones), hacia un aceptor de electrones con un potencial de óxido-reducción muy positivo (alta afinidad por los electrones). En estas condiciones el proceso es termodinámicamente favorable ( $\Delta \mathrm{G}$ total negativo), es decir, libera energía que puede ser utilizada en la realización de algún tipo de trabajo como la síntesis de moléculas altamente energéticas (ATP: Adenosín trifosfato). Esta energía puede ser posteriormente empleada en procesos que tienen que ver con la síntesis de estructuras, locomoción, transporte de sustancias, entre otras.

Similarmente, en una pila voltaica se genera energía mediante el mismo principio físico. Allí también ocurre una transferencia de electrones desde un donador con un potencial de óxido-reducción muy negativo (por 
NOVA - PUBLICACIÓN CIENTÍFICA - ISSN:1794-2470 VOL.4 No. 6 JUNIO - DICIEMBRE DE 2006:1-114

ejemplo $\left.\mathrm{Zn}^{\circ}-0,76 \mathrm{~V}\right)$, hacia un aceptor con un potencial de óxido-reducción muy positivo (por ejemplo $\mathrm{Cu}^{2+}$ $+0,34 \mathrm{~V})$. Una pila es por tanto fuente de energía útil que puede ser empleada por el hombre en infinidad de aplicaciones, Figura 1.

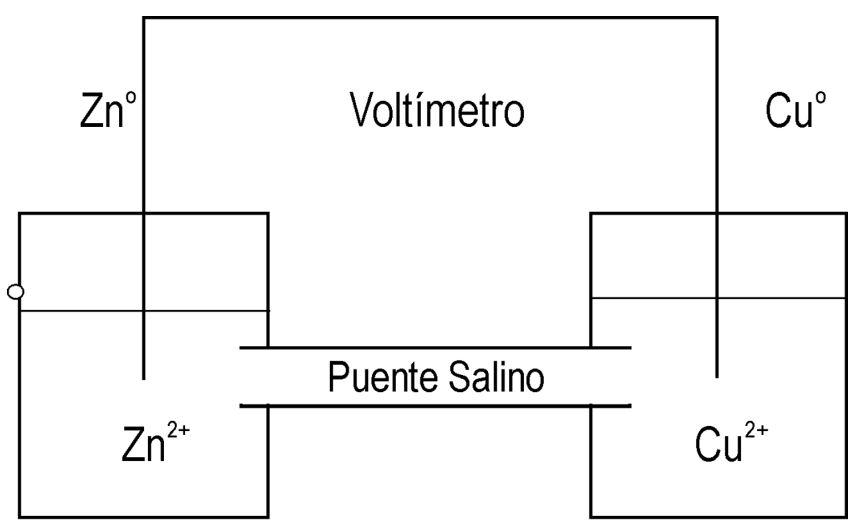

(a)

(b)

Figura 1. Pila voltaica. Los recipientes (a) y (b) contienen soluciones acuosas de iones $\mathrm{Zn}^{2+}$ e iones $\mathrm{Cu}^{2+}$, respectivamente; en los cuales se encuentran inmersos electrodos de $\mathrm{Zn}^{\circ}$ y de $\mathrm{Cu}^{\circ}$. Los electrones fluyen desde el electrodo de $\mathrm{Zn}^{\circ}$ hasta los iones de $\mathrm{Cu}^{2+}$ del recipiente (b). La energía producida es medida mediante el voltímetro. El puente salino conecta los dos recipientes y mantiene el equilibrio de iones.

Todos los organismos vivos emplean las reacciones de oxidación-reducción termodinámicamente favorables para generar la energía necesaria. Los organismos quimiótrofos emplean un sustrato reducido como fuente de energía (y de electrones) con un potencial de oxidoreducción muy negativo (o relativamente poco positivo); y un sustrato oxidado como aceptor de electrones con un potencial de oxido-reducción muy positivo (o relativamente poco negativo). En este proceso por tanto, el sustrato reducido fuente de energía es finalmente oxidado y el sustrato oxidado aceptor de electrones es finalmente reducido.

La mayoría de los organismos quimiorganótrofos (o por lo menos los más conocidos) utilizan como fuente de energía sustancias orgánicas como carbohidratos, lípidos o proteínas. Escherichia coli es una bacteria quimiorganótrofa que puede vivir en un medio enriquecido en glucosa $\left(\mathrm{C}_{6} \mathrm{H}_{12} \mathrm{O}_{6}\right)$. Si existe abundante oxígeno $\left(\mathrm{O}_{2}\right)$ en el medio, realizará respiración aeróbica de la glucosa.
En estas condiciones, la glucosa es oxidada completamente hasta dióxido de carbono y agua, en donde la fuente de energía (y de electrones) es la glucosa y el aceptor final de electrones es el oxígeno [1]. La glucosa posee un potencial de oxido-reducción de $-0,43 \mathrm{~V}$ y el oxígeno, un potencial de oxido-reducción de $+0,82 \mathrm{~V}$. La transferencia de electrones desde la glucosa hasta el oxígeno es entonces un proceso termodinámicamente favorable, con liberación de energía que es empleada por la bacteria en la realización de sus funciones. De maneras similares, otros organismos quimiorganótrofos como hongos, levaduras, protozoos y todos los animales incluyendo el hombre, obtienen la energía que necesitan.

$\mathrm{C}_{6} \mathrm{H}_{12} \mathrm{O}_{6}+6 \mathrm{O}_{2} \rightarrow 6 \mathrm{CO}_{2}+6 \mathrm{H}_{2} 0$ [1] Los organismos quimiolitótrofos utilizan el mismo principio físico ya descrito. Sulfolobus acidocaldarius oxida azufre elemental $\left(\mathrm{S}^{\circ}\right)$ en presencia de oxígeno hasta obtener sulfato $\left(\mathrm{SO}_{4}^{2-}\right)$ [2]. El potencial de óxido-reducción del azufre es $-0,19 \mathrm{~V}$. Igualmente, la transferencia de electrones desde el azufre hasta el oxígeno es un proceso termodinámicamente favorable, con liberación de energía. Nitrobacter es un grupo de bacterias nitrificantes que oxida nitrito $\left(\mathrm{NO}_{2}^{-}\right)$a nitrato $\left(\mathrm{NO}_{3}^{-}\right)$en presencia de oxígeno, con liberación de energía [3]. Aunque el nitrito posee un potencial de oxido-reducción positivo $(+0,42 \mathrm{~V})$, este es menos positivo que el de el oxígeno; ocurriendo de todas formas una transferencia de electrones termodinámicamente favorable. La consecuencia de tener un potencial positivo y cercano al del oxígeno aunque menor, es un crecimiento poblacional relativamente lento. Situación similar ocurre en Thiobacillus ferrooxidans en donde el ión ferroso $\left(\mathrm{Fe}^{2+}\right)$ es oxidado a férrico $\left(\mathrm{Fe}^{3+}\right)$ en presencia de oxígeno [4], con un potencial de óxidoreducción de $+0,76 \mathrm{~V}$.

$$
\begin{aligned}
& \mathrm{S}^{\mathrm{o}}+\mathrm{H}_{2} \mathrm{O}+1 \frac{1}{2} \mathrm{O}_{2} \rightarrow\left(\mathrm{SO}_{4}\right)^{-2}+2 \mathrm{H}^{+}[2] \\
& \left(\mathrm{NO}_{2}\right)^{-}+2 \mathrm{H}^{+}+\mathrm{O}_{2} \rightarrow\left(\mathrm{NO}_{3}\right)^{-}+\mathrm{H}_{2} \mathrm{O} \text { [3] } \\
& \mathrm{Fe}^{2^{+}}+2 \mathrm{H}^{+}+\frac{1}{2} \mathrm{O}_{2} \rightarrow \mathrm{Fe}^{3^{+}}+\mathrm{H}_{2} \mathrm{O}
\end{aligned}
$$


Existen organismos también quimiorganótrofos que han sido reconocidos tan sólo en los últimos años por sus habilidades para biorremediar, es decir, liberar ambientes contaminados con petróleo, insecticidas y herbicidas. Son todos ellos microorganismos que pueden emplear como fuente de energía hidrocarburos tanto alifáticos como aromáticos, sustancias que para la mayoría de las especies que habitan el planeta son tóxicas. Entre ellos es posible encontrar algunas levaduras y algunos géneros de bacterias como Nocardia, Pseudomonas y Mycobacterium. A pesar de lo exótico en su forma de alimentación, la estrategia para obtener energía en estos organismos es la misma; el sustrato reducido (que puede ser propano, butano y hasta octano inclusive) es progresivamente oxidado hasta ser convertido en un intermediario de las vías metabólicas centrales, en este caso ácidos carboxílicos; los cuales siguen siendo oxidados completamente hasta $\mathrm{CO}_{2}$ mediante $\beta$-oxidación, ciclo de Krebs y fosforilación oxidativa (2). El aceptor final de electrones es también el oxígeno [5]. Igualmente, la transferencia de electrones es termodinámicamente favorable con liberación de energía.

$\mathrm{CH}_{3}-\left(\mathrm{CH}_{2}\right)_{6}-\mathrm{CH}_{3}+\frac{25}{2} \mathrm{O}_{2} \rightarrow 8 \mathrm{CO}_{2}+9 \mathrm{H}_{2} \mathrm{O}[5]$

El caso de los fotótrofos es un poco diferente, sin embargo, el principio físico de obtención de la energía necesaria para la síntesis de ATP es el mismo. Existe igualmente un donador de electrones que puede ser moléculas de agua $\left(\mathrm{H}_{2} \mathrm{O}\right)$, sulfuro de hidrógeno $\left(\mathrm{H}_{2} \mathrm{~S}\right)$, azufre elemental $\left(\mathrm{S}^{\circ}\right)$, o iones ferrosos $\left(\mathrm{Fe}^{2+}\right)$. El aceptor de electrones es generalmente moléculas de nicotín adenín dinucleótido fosfato oxidadas $\left(\mathrm{NADP}^{+}\right)$. En el caso de la fotosíntesis oxigénica, el donador de electrones es el $\mathrm{H}_{2} \mathrm{O}$, con un potencial de óxido-reducción de $+0,82 \mathrm{~V}$, y el aceptor de electrones es el $\mathrm{NADP}^{+}$con un potencial de óxidoreducción de $-0,32 \mathrm{~V}$. El flujo de electrones aquí no es espontáneo; sin embargo, es precisamente la radiación solar, la encargada de proporcionar la energía necesaria para que el proceso se torne termodinámicamente favorable. El mecanismo consiste en lo siguiente: La molécula de agua transfiere electrones a las moléculas de pigmento fotosintético como la clorofila. Estas moléculas de pigmento generalmente poseen un potencial de óxidoreducción más positivo que el del agua; por tanto, el flujo de electrones ocurre sin aporte externo de energía. Sin embargo, el siguiente aceptor en la cadena transportadora de electrones tiene un potencial de óxido-reducción negativo. En este paso, el flujo de electrones no es espontáneo. Para que el flujo de electrones continúe es necesario que la radiación solar, active las moléculas de pigmento de tal forma que su potencial de óxido-reducción pase de ser muy positivo a muy negativo. De esta manera, el pigmento fotosintético activado, está en capacidad de continuar la transferencia de electrones termodinámicamente favorable, en la cuál es factible obtener una cantidad de energía suficiente para la síntesis de moléculas de ATP, Figura 2.

De acuerdo con los análisis hechos, es posible concluir que evidentemente los organismos son verdaderas pilas voltaicas vivientes; en donde ocurre una transferencia de electrones desde un donador con un potencial de oxido-reducción muy negativo (o poco positivo) hasta un aceptor con un potencial de oxido-reducción muy positivo (o poco negativo). Esta transferencia de electrones termodinámicamente favorable, libera una buena cantidad de energía útil para realizar trabajo como por ejemplo, en el caso de organismos vivos, la síntesis de moléculas de ATP.

La trascendencia de la conclusión anterior radica en la posibilidad de simplificar la mayoría de los procesos bioquímicos, en este caso el metabolismo energético, en uno a más principios físicos básicos que rigen tanto la materia viva como la inerte. La ganancia de la simplicidad es poder analizar un evento (en este caso biológico) presentado en situaciones muy diferentes (diversidad genética que conlleva a la diversidad metabólica y por tanto a la diversidad de ambientes habitados) pero interpretado desde la misma perspectiva.

Los ejemplos presentados para los organismos quimiótrofos, contemplan siempre como aceptor final de los electrones al oxígeno; sin embargo, no es el único aceptor final de electrones disponible. Es posible encontrar organismos con respiración anaerobia (proceso que difiere ampliamente de la fermentación) y que utilizan a diferencia del oxígeno, aceptores como iones nitrato $\left(\mathrm{NO}_{3}{ }^{-}\right)$, sulfato $\left(\mathrm{SO}_{4}{ }^{2-}\right)$, hierro férrico $\left(\mathrm{Fe}^{3+}\right)$, azufre elemental $\left(\mathrm{S}^{\circ}\right)$ y dióxido de carbono $\left(\mathrm{CO}_{2}\right)$. Podría decirse 


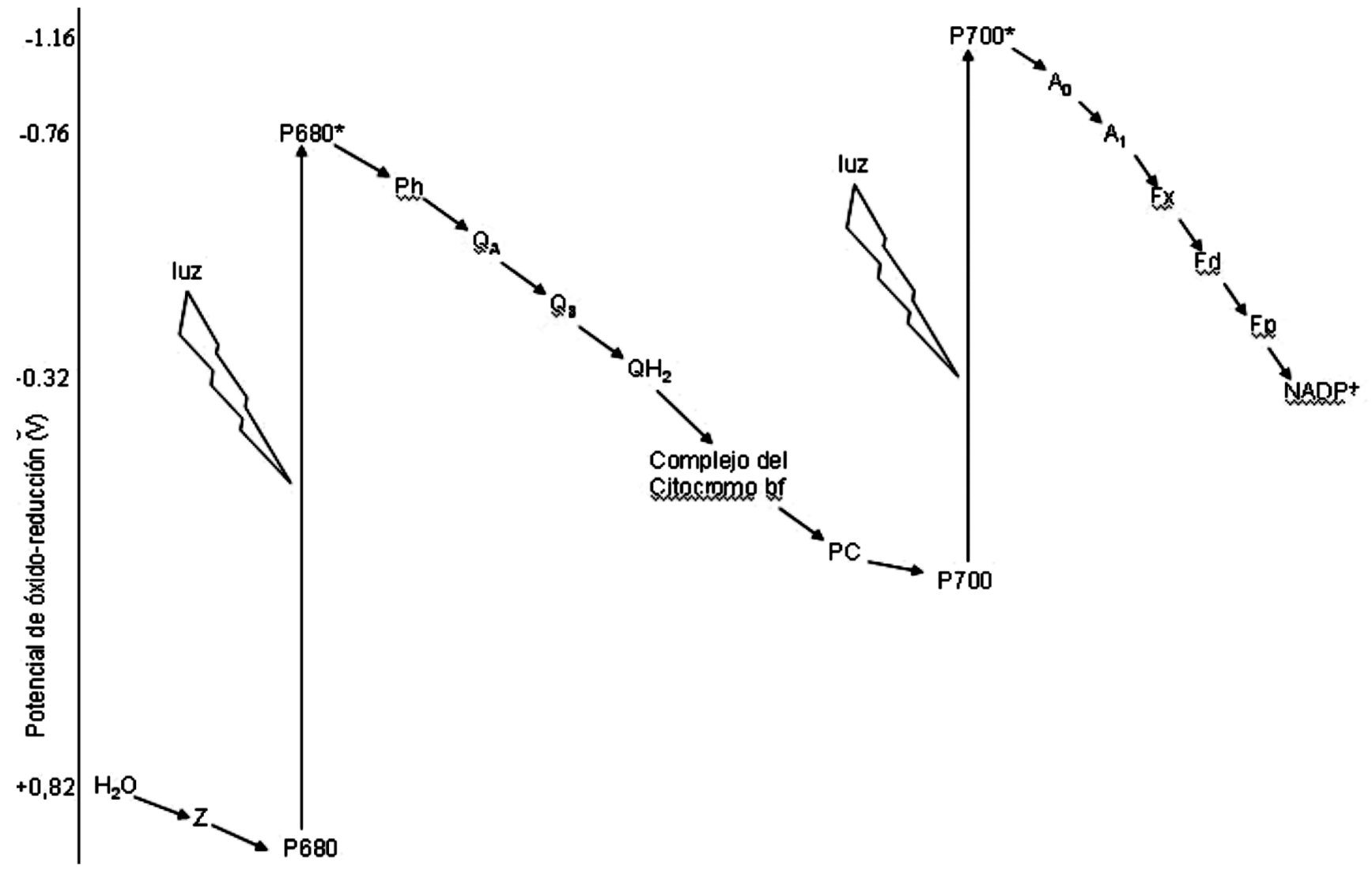

Figura 2. Fotosíntesis oxigénica en una planta. El eje vertical hacia la izquierda del diagrama, representa la variación en los potenciales de óxido-reducción que van desde los muy positivos (abajo) hasta las muy negativos (arriba); de tal manera que la transferencia de electrones es termodinámicamente favorable (con liberación de energía útil) si se realiza desde una molécula que se encuentre en la parte de superior de el diagrama, hasta una molécula que se encuentra en la parte inferior del mismo. El donador inicial de electrones es el $\mathrm{H}_{2} \mathrm{O}$ y el aceptor final es NADP+. Note que el flujo de electrones total no sería termodinámicamente favorable por cuanto el potencial de óxido-reducción del $\mathrm{H}_{2} \mathrm{O}$ es muy positivo (alta afinidad por los electrones) con respecto al potencial del NADP+ que es muy negativo (baja afinidad por los electrones). Sin embargo, la incidencia de la radiación solar, proporciona la energía suficiente para que el pigmento P680 pase a un estado activado (P680*) con un potencial de óxido-reducción tan negativo, que es capaz de generar un flujo de electrones termodinámicamente favorable entre los transportadores de electrones $\mathrm{Ph}, \mathrm{Q}_{\mathrm{A}}, \mathrm{Q}_{\mathrm{B}}, \mathrm{QH}_{2}$, Complejo del citocromo bf y PC. El mismo mecanismo de activación y transporte ocurre en el pigmento P700, permitiendo la reducción final del NADP+, utilizando los transportadores de electrones $A_{0}, A_{1}, F x, F d$ y Fp.

literalmente que estos organismos no respiran oxígeno sino nitrato, sulfato, etc. Nuevamente, la condición para ser aceptor final de electrones es poseer un potencial de óxido-reducción muy positivo (o relativamente poco negativo) con respecto al potencial de óxido-reducción del donador de electrones.

Sin descartar obviamente la necesidad de la maquinaria estructural y funcional de la célula indispensable para que donador y aceptor puedan efectuar el flujo de electrones con liberación de energía, y esta ser canalizada a su vez en la síntesis de moléculas de ATP; el metabolismo energético de cualquier organismo vivo requiere por tanto como condición mínima la existencia de al menos un donador de electrones como fuente de energía, y de al menos un aceptor final de electrones.

El simplificar los procesos en uno o mas principios, permite la evolución de los aspectos conceptuales de una disciplina; fortaleciendo su estructura al agrupar en uno sólo, fenómenos que antes parecían no tener relación entre sí. De esta forma se abre una nueva visión de la bioquímica metabólica; en la que es posible abordar el estudio del metabolismo energético de todos los organismos como pilas voltaicas vivientes, independientemente de los sustratos empleados como fuente de energía (electrones) o de las sustancias empleadas como aceptores de los mismos. La aparente confusión de metabolismos 
diversos, expuesta por organismos que habitan hasta los lugares más extremos, es aclarada si se considera el mismo principio básico subyacente.

El descubrimiento de nuevos seres, la gran mayoría de ellos procariotas, con metabolismos que hasta hace sólo unas décadas podrían parecer inimaginables, ha expandido el horizonte de la bioquímica metabólica. El hallazgo de organismos vivos a $500 \mathrm{~m}$. de profundidad bajo el nivel del mar en fuentes termales marinas a muy altas temperaturas (3), o en ambientes con temperaturas bajo cero, en ausencia de oxígeno y utilizando como aceptores de electrones óxidos de manganeso (4); ha hecho cambiar el concepto que hasta hace muy poco se tenía sobre las condiciones en las cuales la vida sería posible (5). Algunos de estos organismos no sólo utilizan como única fuente de electrones sustancias inorgánicas, sino que además su crecimiento es inhibido por la presencia de moléculas de piruvato (6).

Los organismos eucariotas acapararon durante mucho tiempo casi toda la atención de la comunidad científica. En términos de Nealson y Conrad, el estudio de la vida en la tierra estaba centrado en el estudio de los organismos eucariotas. Hoy en día con el descubrimiento de la gran diversidad genética y metabólica de las células procariotas, la comunidad científica ha volcado sus ojos hacia este otro mundo.

El hallazgo de nuevas formas de vida encontradas en procariotas ha permitido ampliar la estructura conceptual que explica el metabolismo energético, no centrado exclusivamente en la utilización de sustratos orgánicos clásicos como carbohidratos, lípidos o proteínas y en el aparentemente universal oxígeno como aceptor final de electrones.

Lamentablemente este amplio panorama que ha sido desvelado, no es reflejado en el sistema educativo. La bioquímica que hoy en día es enseñada en las instituciones superiores es la bioquímica del mundo eucariota, un mundo que como se ha dicho, es bastante limitado comparado con la riqueza metabólica que ofrece la diversidad procariota. Inclusive los textos de bioquímica de mayor prestigio $(7,8,9)$ tienen alusiones pobres o ninguna a otros sistemas vivientes diferentes a los del mundo eucariota; asumen la obtención de energía únicamente a partir de carbohidratos y lípidos, y se habla de respiración (no se aclara si es aerobia o anaerobia porque si se desconoce la anaerobia, no es necesario aclararlo) y fermentación (que en la mayoría de los casos es confundida con respiración anaerobia). Se asume por defecto que todos les organismos poseen organelos internos y se explicita muy bien las reacciones ocurridas en la mitocondria; pero no se da ninguna información que permita entender cómo se las arregla un organismo que carece de ella.

La situación es más preocupante cuando en algunos casos la visión de bioquímica a enseñar recuerda todavía la vieja idea medieval del antropocentrismo. Salvo en los cursos de bioquímica ofrecida para estudiantes de medicina y demás ciencias de la salud, no es clara la tendencia absolutamente sesgada a enseñar únicamente la bioquímica humana, como ha ocurrido en muchos cursos de bioquímica ofrecidos para estudiantes de biología, química, microbiología y ciencias afines.

Aunque es cierto que muchos de los descubrimientos son recientes y es comprensible que el cambio no se haya dado, en otras circunstancias no hay justificación. Se conoce de la existencia de organismos quimiolitótrofos desde finales del siglo XIX, mediante el aporte de Winograsdsky, citado por Madigan, quien encontró que precisamente existían organismos cuya única fuente de energía es inorgánica. Si estos hallazgos datan del siglo XIX; ¿cuál es la razón que amerita que en el siglo XXI aún no sean incorporados en la estructura conceptual de la nueva bioquímica y por ende en la estructura mental de profesores y estudiantes? ¿Cuál es el temor que se le tiene a observar la gran diversidad que la Naturaleza ofrece, aun cuando es posible aplicar principios físicos universales que logran explicar, como es el caso en cuestión, el metabolismo energético de los seres vivos? ¿Acaso no resulta demasiado sencillo asumir los organismos vivos como pilas voltaicas en donde basta averiguar por el donador y por el aceptor de electrones para identificar el tipo de metabolismo que cada organismo posee? 
Sea esta discusión una invitación al cambio y a la realización de propuestas que lleven a re-escribir los libros de texto de bioquímica en los que sea plasmada la maravillosa diversidad de la naturaleza.

\section{Referencias}

1. Madigan, M; Martinko J y Parker J. In Brock Biología de los Microorganismos. Décima edición. Prentice Hall Iberia. Madrid. 2003. 1011 Págs.

2. Parés, R. y A. Juárez. Bioquímica de los microorganismos. Editorial Reverté Barcelona. 1997. 380 Págs.

3. Guerrero, R. Crucial crises in biology: life in the deep biosphere. International Microbiology. 1998. (1) 285-294.
4. Nealson, K y Saffarini, D. Iron and manganese in anaerobic respiration: environmental significance, physiology and regulation. Annual Review of Microbiology. 1994 (48) 311-343.

5. Nealson, K y Conrad, P. Life: past, present and future. Philosophical Transactions of Royal Society of London. 1999. 354: 1923-1929.

6. Parshina, S; Kijlstra, S; Henstra, A; Sipma, J; Plugge, C and Stams, A. Carbon monoxide conversion by thermophilic sulfate-reducing bacteria in pure culture and in co-culture with carboxythermus hydrogenoformans. Applied Microbiology and Biotechnology. 2005 (68) 390-396.

7. Harvey, R; Champe, P. Bioquímica. Tercera Edición. Editorial Mc. Graw Hill. México. 2006.

8. Mathews, C; Van Holde, K; Ahern, K. Bioquímica. Pearson educación S.A. Madrid. 2002.

9. Nelson, D, Cox, M. Lehninger Principles of Biochemistry. Worth Publishers. New York. 2004. 1120 págs. 